\title{
Dose Adjustment
}

National Cancer Institute

\section{Source}

National Cancer Institute. Dose Adjustment. NCI Thesaurus. Code C82495.

An indication or description of a change in dosage. 